\title{
CALCULATING FRICTIONAL LOSSES IN BELLEVILLE SPRINGS BY LINEAR INTERPOLATION
}

\author{
Dipesh Maharjan ${ }^{1}$, Shaheen Shah $^{2}$, Abdelsalam Abugharara ${ }^{3}$, Stephen Butt ${ }^{4}$ \\ Faculty of Engineering and Applied Science \\ Memorial University of Newfoundland, St. John's, NL, CA \\ 12dmaharjan@mun.ca, ${ }^{2}$ mshaheens@mun.ca, ${ }^{3}$ a_nasar@mun.ca, ${ }^{4}$ sdbutt@mun.ca
}

\begin{abstract}
The use of Belleville springs has been proven to be beneficial in tackling the problems related to elastic interaction, creep, differential thermal expansion or in the isolation of seismic vibrations in bolted joints. Because of its high and easily variable spring rates, the use of these springs can also be observed in passive vibration assisted rotary drilling (VARD) tools. Because of relative movement of spring with respect to supporting surfaces and the mating spring surfaces, frictional losses in the spring take place during each compression and relaxation cycle leading to a slightly different load-deflection curve from what has been defined in the literature. The geometry of the spring combined with different stacking configurations complicate the study of frictional losses in these kinds of springs.

This work presents a new method to calculate the displacement of different points of Belleville springs during its loading and unloading using linear interpolation method. The results of spring displacement are then used to develop a model to calculate frictional load as a function of spring deflection, which is used to analyse load-deflection curves of springs with different dimensional and frictional parameters. The developed methodology is used to plot and understand characteristics curves of four different kind of Belleville springs; High Load, Standard, Force Limiting and Force Adjusting Belleville springs by plotting the graphs for different free-height to thickness and diameter ratios. In the later section of the paper, the proposed methodology has been used to visualize the load-deflection characteristics of Belleville springs used in the p-VARD tool of the Large Drilling Simulator, one of the state-of-the-art drilling simulators at Memorial University of Newfoundland. Results show that understanding of the characteristic curves of different spring configuration helps to plan to drill with desired WOB using a p-VARD tool.
\end{abstract}

Keywords: Belleville spring, Frictional loss, loaddeflection curve, $p$-VARD springs

\begin{tabular}{ll} 
& \multicolumn{1}{c}{ NOMENCLATURE } \\
$\mathrm{t}$ & spring thickness \\
$\mathrm{r}_{1}$ & spring inside radius \\
$\mathrm{r}_{2}$ & spring outside radius \\
$\mathrm{H}$ & total height \\
$\mathrm{h}$ & free height \\
$\varnothing$ & spring angle \\
$\theta$ & angle of neutral line \\
$\delta$ & spring deflection \\
$\mathrm{P}$ & spring load \\
$\mathrm{E}$ & Young's modulus \\
$\vartheta$ & Poisson's ratio \\
$\mathrm{I}$ & number of springs in series \\
$\mathrm{J}$ & number of springs in parallel \\
$\mathrm{u}_{\mathrm{s}}$ & surface sliding displacement \\
$\mathrm{u}_{\mathrm{e}}$ & edge displacement \\
$\mu_{\mathrm{s}}$ & surface friction factor \\
$\mu_{\mathrm{e}}$ & edge friction factor
\end{tabular}

\section{INTRODUCTION}

Belleville spring, patented and named after the inventor Julien Belleville in France in 1867 is one of the frequently used springs to support very large load with a small installation space. Although many works have been performed for better understanding of the load-deflection curve of the Belleville springs, the first equation proposed by Almen-Lazlo [1] is the most cited and tested approach by several researchers and laboratories including General Motors Corporation. AlmenLaszlo's equation was dependent on three major assumptions:

1) Small angular deflection takes place at the spring crosssection.

2) Spring deflection takes place by mere rotation about a neutral point.

3) Loads are concentrically distributed, and radial stresses are negligible.

Researches which followed were mostly centered around the modification of the basis assumptions of Almen-Laszlo's theory [2]. In a paper by Rosa et. al. 2001 [3], they have based their discoveries on the ground that the radial stresses are 
linked to the tangential stresses by the equilibrium equation and designed a spring with variable thickness to obtain wide range of constant force over a range of deflection. Similarly, with the development of experimental and simulation tools, new researches focused on the numerical analysis and FEA simulations of Belleville springs. In one of the works by Karakaya [4] the effect of material non-linearity was studied to enable the manufacturing of springs with composite material instead of conventional steel. Simulation techniques have made it possible to study the effect of tapered-ness in the spring design. Results from the work by Rosa et. al. [3] have shown possibility of manufacturing springs that are radially tapered thus increasing the linear region of the load-deflection curve, meaning that springs can be operated with higher range of constant loads. Apart from these, studies have also been done to study the effect of having slotted Belleville springs [5].

Although much work has been done in the field of Belleville springs, study of effect of friction in Belleville springs is very limited. Curti and Montanini [5] in their approach to generate more accurate spring characteristics studied the effect of friction between spring edge and the supporting surface. The theoretical formulation was verified by comparing the results with that from the FEA. However, their work was limited to the study of friction between the spring and supporting surfaces only. This limited the implementation of this work as Belleville springs are always stacked in series, parallel or the combination of both.

In a work by Ozaki et. al. [6], in addition to the edge friction between the spring and the supporting surfaces, the surface friction that exists between the mating surfaces of two or more parallelly stacked springs was also incorporated. The authors have shown a comparison between Almen-Laszlo's equation and the results from the FEA which clearly show the missing frictional component in Almen-Laszlo's equation during loading and unloading of the springs, thus highlighting the need of more research in this field. They use a link type connection that neglects the spring rotational moment to calculate the displacement of spring edge with respect to the supporting surface and the displacement of spring surfaces of parallelly stacked springs. The hysterics loss during one spring cycle is then calculated in parts as a summation of frictional loss at the edges and that on the spring surfaces. The work further extends to formulate a generic equation which can be used to determine the hysterics losses during loading and unloading of the springs.

In this paper, a new approach of interpolation is used to calculate the relative displacements that takes place in the springs during its loading and unloading cycle. The methodology proposed in this paper stands as an alternative to the methodology of rotational matrix proposed by Ozaki et. al. [6] for calculating the displacement components of different points in the spring. In the sections that follow, calculation of spring displacements is presented in section II. This section also provides a breakdown for the derivation of edge friction loss and surface friction loss. In section III, the characteristics curves are generated for different free-height to thickness ratios and diameter ratios followed by the study of different spring configuration of LDS p-VARD tool in section 5. The basic trends that are identified in the plotted results are discussed on more detail in section $\mathrm{V}$ along with some concluding remarks.

\section{Calculation Of SPRING DisPlacements}

This section describes the proposed methodology to calculate the displacement of spring edge with respect to the supporting surface and the relative displacement of the mating surfaces of springs stacked in parallel. The derivations made in this section of the report are based upon the fundamental assumptions of Almen-Laszlo's work, i.e. the cross-section of the spring doesn't deform but rather rotates about a neutral point when it is deformed. This deformation phenomenon is illustrated in Figure 1.

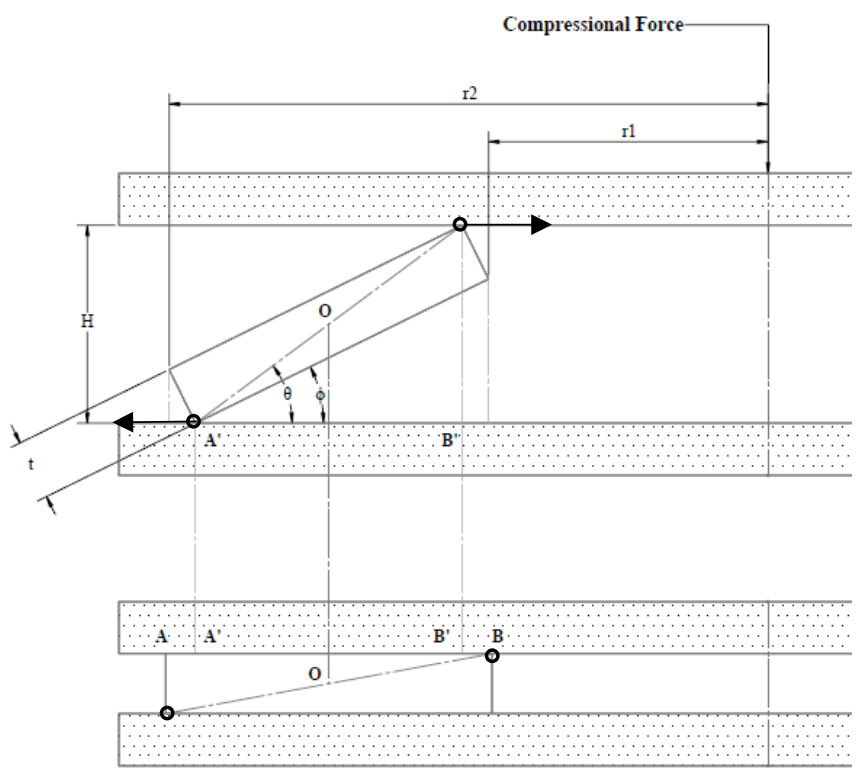

Figure 1: Showing rotation of spring cross-section about the neutral point during compression

It can be seen from Figure 1 that when the spring is compressed, the points which are in contact with the supporting surfaces will slide equal distances in transverse directions, without any deformation in the spring crosssection. Moreover, the distance travelled by the top point is equal and opposite to the distance travelled by the lower point. When the spring is fully compressed, the total transverse displacements of these points in opposite direction can be estimated using below formula.

$$
\begin{aligned}
\text { displacement of lower edge }\left(A A^{\prime}\right) \\
=\text { displacement of top edge }\left(B B^{\prime}\right) \\
=\frac{\frac{t}{\tan (\theta-\emptyset)}-r_{2}+r_{1}+2 t \sin \emptyset}{2}
\end{aligned}
$$


Where $\emptyset$ and $\theta$ are the initial inclinations of the spring and neutral line with the horizontal. The angles $\emptyset$ and $\theta$ are related to spring parameters by below relations.

$$
\begin{gathered}
\tan \emptyset=\frac{H-t \cos \emptyset}{r_{2}-r_{1}-t \sin \varnothing} \\
\theta=\tan ^{-1} \frac{H}{r_{2}-r_{1}-2 t \sin \emptyset}
\end{gathered}
$$

The free height $(h)$, which is also equal to the maximum deflection of the spring can be estimated using below formula.

$$
h=H-t \cos \varnothing
$$

The next step after determining the initial and the final condition is to interpolate the values of lateral displacement as a function of spring deflection $(\delta)$.

Table 1: Interpolation table for intermediate edge displacement

\begin{tabular}{|c|c|c|c|}
\hline & $\begin{array}{c}\text { Initial } \\
\text { point }\end{array}$ & Final point & $\begin{array}{c}\text { Intermediate } \\
\text { points }\end{array}$ \\
\hline Deflection & 0 & $h=H-t \cos \emptyset$ & $\delta$ \\
\hline $\boldsymbol{u}_{\boldsymbol{e}}$ & 0 & $\frac{t}{\tan (\theta-\varnothing)}-r_{2}+r_{1}+2 t \sin \emptyset$ & $\delta \times \frac{u_{e_{-} \text {final }}}{h}$ \\
\hline
\end{tabular}

A similar concept can be applied for determining the displacement of spring surface with respect to the mating spring surfaces when they are stacked in parallel. Figure 2 shows a schematic diagram of two springs stacked in parallel and subsequently flattened. From the understanding of geometrical relationships of Figure 2, it can be concluded that the mating surfaces of two springs will produce a maximum sliding displacement $\left(u_{s}\right)$ which is equal to $t \sin \varnothing$. Using a similar interpolation method, sliding displacement can be calculated as a function of spring deflection $(\delta)$.

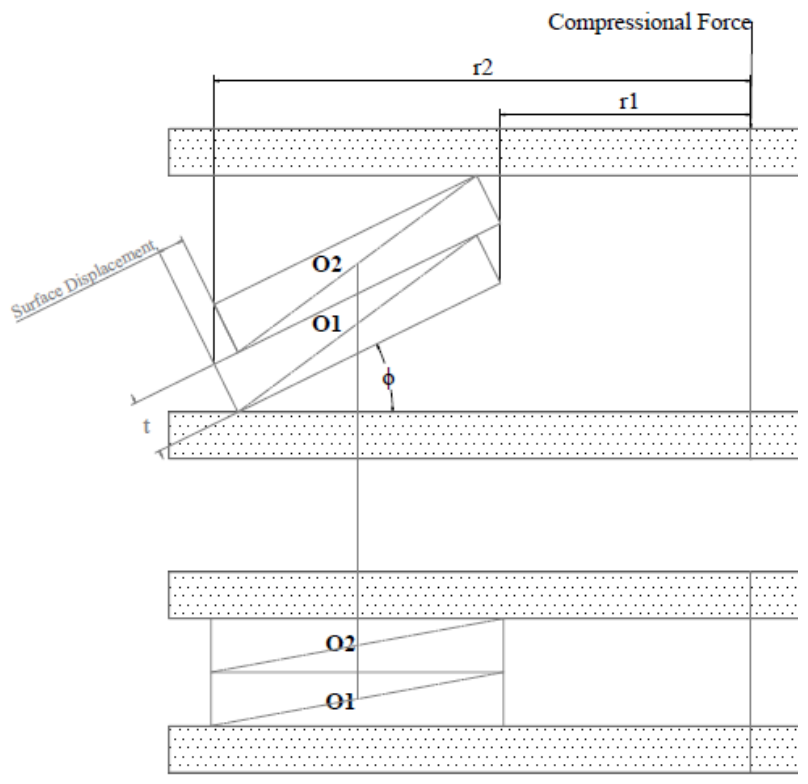

Figure 2: Showing the compression of two springs stacked in parallel
Table 2: Interpolation table for intermediate surface displacement
Initial
Final point
Intermediate points
Deflection
0
$h=H-t \cos \emptyset$
$u_{s}$
0
$t \sin \emptyset$
$t \sin \emptyset \times\left(\frac{\delta}{H-t \cos \varnothing}\right)$

Determining the frictional dissipation during the loading and unloading of the spring is the next objective. Following the methodology proposed by Ozaki et. al. [6], frictional dissipation is calculated as product of frictional force and corresponding displacement, which can then be converted to equivalent load increment by dividing by the equivalent displacement. Thus, corrected load-deflection curve for the Belleville spring as proposed by Ozaki et. al. [6] can be used which undertakes the effect of frictional losses as follows:

$$
\begin{aligned}
& P=J P_{m}+\Delta P \\
& P_{m}=\frac{E \bar{\delta}}{\left(1-\vartheta^{2}\right) r_{2}^{2}}\left[(h-\bar{\delta})\left(h-\frac{\bar{\delta}}{2}\right) \frac{t}{M}+\frac{t^{3}}{N}\right] \\
& \frac{1}{M}=\left[\frac{\gamma+1}{\gamma-1}-\frac{2}{\ln \gamma}\right] \pi\left(\frac{\gamma}{\gamma-1}\right)^{2} \\
& \frac{1}{N}=\frac{\pi}{6} \ln \gamma\left(\frac{\gamma}{\gamma-1}\right)^{2} \\
& \gamma=\frac{r_{2}}{r_{1}} \\
& \bar{\delta}=\frac{\delta}{I} \\
& \Delta P=\frac{\Delta E_{f e}+\Delta E_{f s}}{\Delta \bar{\delta}} \\
& \Delta E_{f e}=2 P_{m} \mu_{e} \Delta \bar{u}_{e} \\
& \Delta E_{f s}=I(J-1) P_{m} \cos \emptyset^{\prime} \mu_{s} \Delta \bar{u}_{s}
\end{aligned}
$$

The notation $\emptyset^{\prime}$ in above equation is different from the angle $\varnothing$ which was defined earlier. $\emptyset^{\prime}$ is the measure of intermediate angle between the spring and the horizontal and can be calculated from interpolation method as a function of spring deflection $(\delta)$.

Table 3: Interpolation table for intermediate spring angle, $\varnothing^{\prime}$

\begin{tabular}{c|c|cc|}
\hline & $\begin{array}{c}\text { Initial } \\
\text { point }\end{array}$ & Final point & Intermediate points \\
\hline Deflection & 0 & $h=H-t \cos \emptyset$ & $\delta$ \\
$\underset{\left(\phi^{\prime}\right)}{\text { Spring angle }}$ & $\varnothing$ & 0 & $\emptyset \times\left(1-\frac{\delta}{H-t \cos \varnothing}\right)$
\end{tabular}


It can be observed from the equations that equivalent displacement $(\bar{\delta})$ is used to calculate the Almen-Laszlo's load approximation and frictional losses instead of total deflection $(\delta)$, where equivalent displacement $(\bar{\delta})$ is the displacement per series stack. This is because when Belleville springs are stacked together, I number of springs in series stacking multiplies the total deflection by $I$ and $J$ number of springs in parallel stacking multiples the total load by $J$ [7]. Thus, when the total deflection of the spring stack is $\delta$, deflection of each series stack will be $\bar{\delta}=\delta / I$.

\section{RESULTS}

So far, the methodology to calculate the displacement of different points of spring during deflection has been derived from the simple way of interpolation. The formulae can be extended to calculate frictional dissipation as a function of spring deflection. Figure 3 shows a load-deflection curve obtained from above interpolation method which is similar to that proposed by Ozaki et. al.[2, Figure $9 \mathrm{c}$.

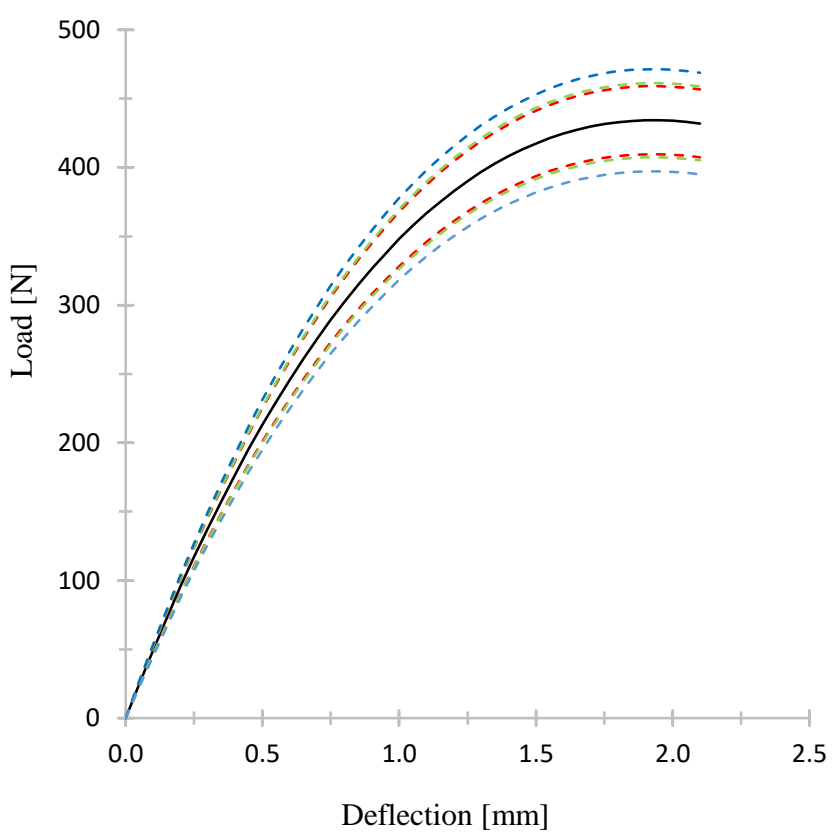

Figure 3: Load-deflection curve generated by proposed method [Spring parameters $(\mathrm{mm}): H=1.7, r_{1}=18.08, r_{2}=24.45, t=0.5, I=3, J=2$ ]

Next, the paper studies of effect of spring parameters on frictional loss in Belleville springs. One of the spring parameters used in specifying the Belleville springs is free height to thickness ratio, $h / t$. A set of $h / t$ ratios $[1,1.4$ and 2.4] were plotted for the $I=2, J=2$ spring configuration with constant edge friction coefficient $\left(\mu_{e}=0.3\right)$, and varying surface friction coefficient $\left(\mu_{s}=0,0.1,0.3\right)$. Different sets of $h / t$ ratios were obtained by keeping total height of the spring constant at $H=1.7 \mathrm{~mm}$ and changing the thickness, $t$. Figure 4 is the graph obtained by plotting the load-deformation diagram of these spring configuration, which is identical to the one plotted by Ozaki et. al [2, Figure 10 a].

Figure 4 can also be used to identify the type of Belleville spring by the nature of its load-deflection curve. As grouped by most of the manufacturers, Belleville springs can be categorized into High Load, Standard, Force Limiting and Force Adjusting Belleville springs [8]. The springs that produce curves similar to $h / t=0.5$ can be categorized as High Load Belleville springs. These springs are very thick and have high load capacity. The load-deflection curve for these springs is mostly linear. Similarly, springs with curves similar to $h / t=1$ are the Standard Belleville springs; these springs are known for their excellent fatigue resistance. Loaddeflection curve of the Standard Belleville springs are linear for small deflections. The third type of spring that can be identified by the load-deflection curve corresponds to $h / t=$ 1.4, Force Limiting Belleville springs. The load-deflection curve for these types of springs is linear when the spring starts to deflect and becomes flat after certain amount of compression. Because of this nature, these types of springs are used in clutches, brakes or PSVs. The fourth category of Belleville spring is the Force Adjusting type. These springs have high $h / t$ ratios, 2.4 in this case. The load-deflection curve for these springs starts out linear, reaches a maximum value and then loses force after certain percentage of deflection.

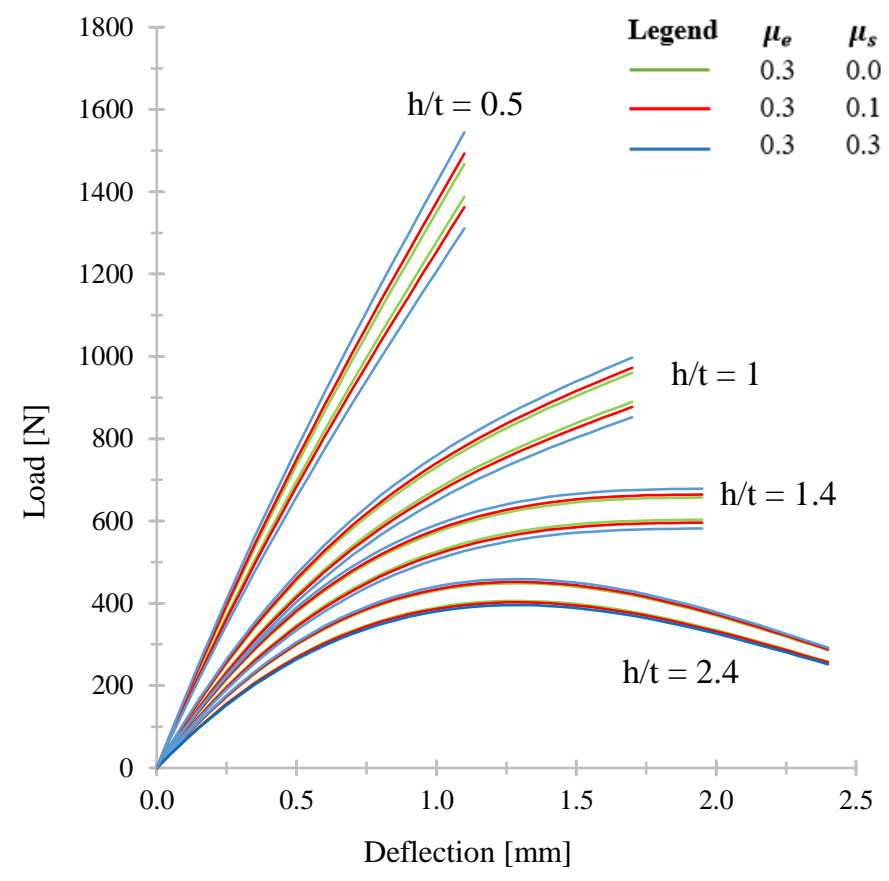

Figure 4: Effect of change of $h / t$ ratio on frictional dissipation [Spring parameters (mm): $H=1.7, r_{1}=18.08, r_{2}=24.45, I=2, J=2$ ]

The effect of diameter ratio, $\gamma$ on the load-deflection curve can be observed in the similar manner. For this, three diameter 
ratios $[\gamma=1.4,1.6$ and 2] were plotted for a spring with of $r_{2}=24.45 \mathrm{~mm}, t=0.5 \mathrm{~mm}$ and $H=1.7 \mathrm{~mm}$. The value of $r_{1}$ was varied to match the corresponding diameter ratio. Figure 5 shows the curves plotted with these scenarios together with the variation in friction coefficients.

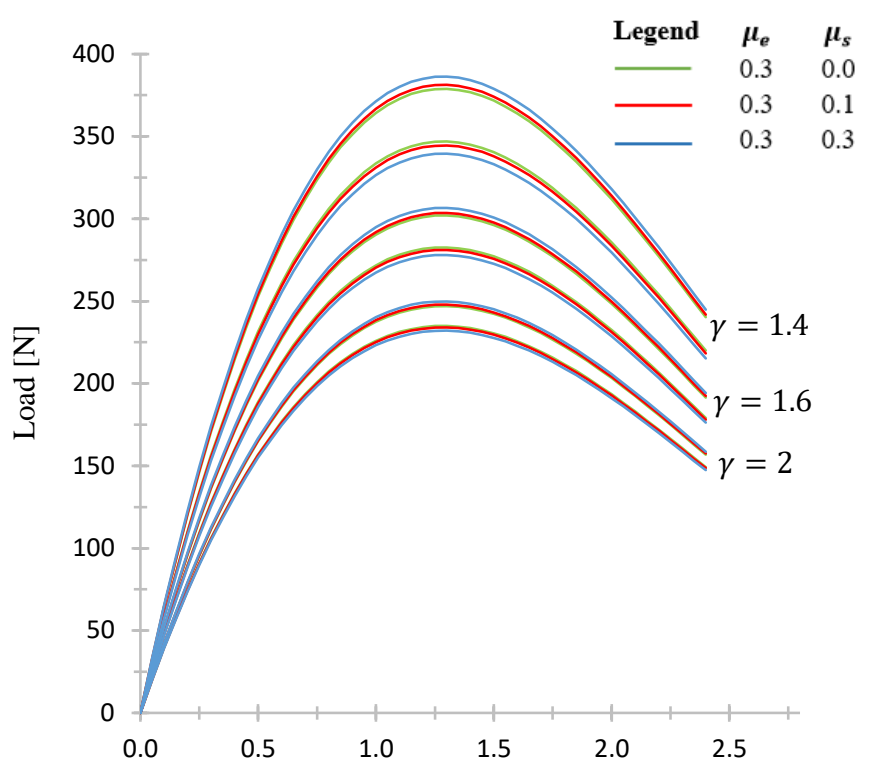

Deflection $[\mathrm{mm}]$

Figure 5: Effect of change of diameter ratio on frictional dissipation [Spring parameters ( $\mathrm{mm}$ ): $H=1.7, r_{2}=24.45, t=0.5, I=2, J=2$ ]

The curves plotted in Figure 5 show a decreasing pattern in frictional losses as the diameter ratio increases. Similarly when the overall pattern of the load-deflection curve is analyzed, it is observed that Belleville springs or stack of Belleville springs tend to become more compliant as the diameter ratio goes on increasing.

\section{ANALYSIS OF LSD P-VARD SPRINGS}

The analysis of the Belleville springs used in LDS pVARD tool comprises of understanding of nature of their characteristics curves and the frictional losses in them. Specifications of the springs that are analyzed is listed in Table 4.

Table 4: Spring Specifications for LSD p-VARD

\begin{tabular}{|c|c|c|c|c|c|}
\hline $\begin{array}{c}\text { Spring } \\
\text { number }\end{array}$ & ID & OD & Thickness & Total height & h/t \\
\hline 1 & $\boldsymbol{d}_{\boldsymbol{l}}(\boldsymbol{i n})$ & $\boldsymbol{d}_{2}(\boldsymbol{i n})$ & $\boldsymbol{t}(\boldsymbol{i n})$ & $\boldsymbol{H}(\boldsymbol{i n})$ & \\
\hline 2 & 1 & 2 & 0.065 & 0.13 & 1.00 \\
\hline 3 & 1 & 2 & 0.084 & 0.136 & 0.62 \\
\hline 4 & 1 & 2 & 0.097 & 0.145 & 0.499 \\
\hline & 1 & 2 & 0.142 & 0.177 & 0.249 \\
\hline
\end{tabular}

Figure 6 shows the load-deflection curves for the $I=2$, $J=2$ configuration of all four springs with constant edge friction coefficient $\left(\mu_{e}=0.3\right)$, and varying surface friction $\operatorname{coefficient}\left(\mu_{s}=0 \& 0.3\right)$. The graph helps to predict how spring deflection is going to vary with increasing/decreasing load, which in the case of p-VARD tool is the drilling weight on bit.

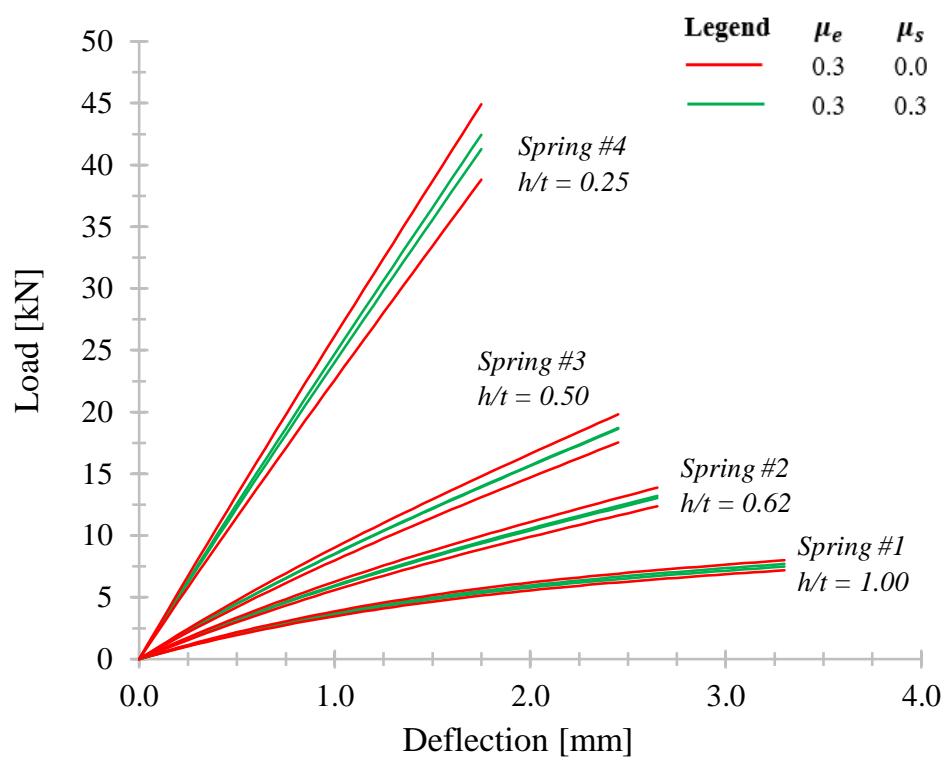

Figure 6: Load-deflection diagram of Belleville springs for LDS p-VARD

The load-deflection curves of Figure 6 can help us determine the nature of spring stiffness exhibited by the Belleville springs. Spring \#1 with $h / t=1.0$ exhibit the characteristics of a Standard Belleville spring i.e. the deflection is proportional to load at the beginning of the stroke only. As the load increases further, the curve tends to become more cubic in nature. Because of this nature of spring \#1, when configuring the p-VARD tool with this spring, care should be taken not to approach the full stroke of the spring compression. Similarly, spring \#2 with $h / t=0.62$ appears to have more linear load-deflection relationship than spring \#1. Spring \#3 and \#4 on the other hand have fully linear load-deflection relationship.

The other goal of the analysis is to study the nature of frictional losses in Belleville springs. From the graphs that have been plotted, it is observed that frictional loss is directly proportional to compressive force. Moreover, surface friction accounts for higher losses than the edge friction as soon as the number of parallel stacking is increased to $J=2$. This phenomenon is observed in all four springs. 


\section{CONCLUSION}

In this work, linear interpolation has been used to calculate the frictional losses in Belleville springs. This method relies on the basis assumptions put forth by Almen-Laszlo in their original work [1] to characterize these springs and on the modification of the work by Ozaki et. al. [6], which was based on the method of rotational matrix. Observation of the results plotted by this method shows that they closely match the ones predicted by Ozaki et. al.

Frictional losses in the springs is dependent on type of spring stacking. When the number of springs stacked in parallel is less, edge friction accounts for most of the frictional loss. This is not hold true when the number of parallel springs is increased, where surface friction becomes more dominant. Similarly, as observed from the graphs, frictional loss is also dependent on the spring load or spring deflection. For the High Load and Standard Belleville springs, frictional loss increases with increase in spring deflection. However, for the Force Limiting Belleville springs, frictional loss becomes constant at the flat region of the load-deflection curve and for the Force Adjusting Belleville springs, frictional loss is maximum at the peak of load-deflection curve and starts to decrease as the spring is compressed more.

It can be observed from the graphs that maximum load of these springs varies as per the type of spring or more parametrically, on the $h / t$ ratio. More predominantly observed in the Force Limiting and Force Adjusting springs, increasing the load beyond the maximum load will cause the springs to lose force or provide steady resistance to deformation. Understanding the characteristic curve and the hysteresis loop of Belleville springs is crucial while designing components involving these springs as selection of spring with inadequate capacity may lead to unsatisfactory performance of the component and/or its failure.

This approach of calculating the frictional losses can be experimentally validated. Based on the theoretical understanding of this phenomenon, it can be predicted that the experimental curve for spring compression will to be similar to the loading curve presented in Figure 3. Variation of the experimental results from this theory, if any, can be used in calibrating this model to improve or validate its accuracy.

Similarly, as a further step to calibrate the p-VARD tool, the $\mathrm{p}$-VARD index can be developed to readily make available the optimal spring configuration that will be able to work at the optimal WOB in drilling experiments. As far as the nature of the load-deflection curve is concerned, changing the spring configuration only scales up/down the values of maximum load and/or maximum deflection while maintaining the original characteristics of a single spring. Calculative selection of spring and spring configuration helps in preventing premature flattening of springs and maintaining desired compliance in the p-VARD tool.

\section{REFERENCE}

[1] J. O. Almen and a. Laszlo, "The uniform-section disk spring," Trans. Am. Soc. Mech. Eng., vol. 58, pp. 305314, 1936.

[2] Y. Zhiming and Y. Kaiyuan, "A study of belleville spring and diaphragm spring in engineering," J. Appl. Mech. Trans. ASME, vol. 57, no. 4, pp. 1026-1031, 1990.

[3] G. La Rosa, M. Messina, and A. Risitano, "Stiffness of variable thickness belleville springs," J. Mech. Des. Trans. ASME, vol. 123, no. 2, pp. 294-299, 2001.

[4] Ş. Karakaya, "Investigation of hybrid and different crosssection composite disc springs using finite element method," Trans. Can. Soc. Mech. Eng., vol. 36, no. 4, pp. 399-412, 2012.

[5] G. Curti and R. Montanini, "On the influence of friction in the calculation of conical disk springs," J. Mech. Des. Trans. ASME, vol. 121, no. 4, pp. 622-627, 1999.

[6] S. Ozaki, K. Tsuda, and J. Tominaga, "Analyses of static and dynamic behavior of coned disk springs: Effects of friction boundaries," Thin-Walled Struct., vol. 59, no. January, pp. 132-143, 2012.

[7] M. Paredes and A. Daidié, "Optimal catalogue selection and custom design of belleville spring arrangements," Int. J. Interact. Des. Manuf., vol. 4, no. 1, pp. 51-59, 2010.

[8] "Types of Belleville Springs, Belleville International" Load vs. Deflection. [Online]. Available: https://bellevilleintl.com/engineering/load-vs-deflection/. [Accessed: 30-Nov-2019]. 